\title{
Cost-Effectiveness of Allergen Immunotherapy
}

\section{Cristoforo Incorvaia ${ }^{1 *}$, Patrizia Berto ${ }^{2}$, Renato Ariano ${ }^{3}$, Rita Elia ${ }^{1}$ and Franco Frati ${ }^{4}$}

${ }^{1}$ Allergy/Pulmonary rehabilitation Unit, ICP Hospital, Milan, Italy

${ }^{2}$ Pbe consulting, Verona, Italy

${ }^{3}$ Internal Medicine Department, Bordighera Hospital, ASL 1, Imperia, Italy

${ }^{4}$ Medical and Scientific Department, Stallergenes, Milan, Italy

\begin{abstract}
The current burden of allergic diseases, considering both direct and indirect costs, is very relevant. In fact the estimated cost for allergic rhinitis (AR) is 4-10 billion dollars/year in the US and averages an annual cost of 1089 euros per child/adolescent and 1543 euros per adult in Europe. The cost is obviously higher when including also allergic asthma. Strategies aimed at reducing the clinical severity of allergy are therefore most relevant from both a societal and healthcare system's perspective. Among them, allergen immunotherapy (AIT) showed preventive capacity and also a carryover effect once treatment is discontinued, thus further reducing the costs. A number of studies demonstrated a favorable cost-benefit ratio for AIT. The first studies in the 1990s evaluating subcutaneous AIT in patients with allergic rhinitis and asthma, reported significant reductions of direct and indirect costs in subjects treated with AIT, as compared to those treated with symptomatic drugs. This was fully confirmed in recent studies conducted in European countries, also including sublingual immunotherapy, as well as in studies conducted in the US. In particular, the conclusion of a recent study on health care cost benefits of AIT in children with AR suggesting that "Greater use of this treatment in children could significantly reduce AR-related morbidity and its economic burden" should be kept in mind when considering the optimal choice of medical treatment in patients with AR or asthma.
\end{abstract}

Keywords: Allergen immunotherapy; Drug treatment; Rhinitis; Asthma; Pharmacoeconomics

Abbreviations: AR: Allergic Rhinitis; AIT: Allergen Immuno Therapy; DM: Deutschmarks; DKK: Danish Kroner; ICER: Incremental Cost-Effectiveness Ratio; NHS: National Healthcare System; ROPP: Retrospective Observation Physician Panel; QALY: Quality Adjusted Life Year; SLIT: Sublingual Immunotherapy

\section{Introduction}

Allergic diseases, especially allergic rhinitis (AR), asthma and atopic dermatitis, show increasing prevalence in developed countries with figures up to $20 \%$ [1-4] which characterize them as medical problems of growing importance. High prevalence inevitably carries out also a relevant economic burden: for example, concerning AR, in the United States an increasing number of billion dollars per year is spent both as direct and indirect costs [5-11]. Direct costs are those associated to drug treatment, physician visits and, especially for asthma, to hospital admissions, while indirect costs are related to reduced/missed work productivity [9] and include patient's quality of life, cognitive and learning functions, decision making and self-perception [10].

Yet in early 1990s a study calculated that two-thirds of the total cost of AR in the US- estimated in 1.8 billion dollars- was due to direct medical costs and one third was due to indirect costs [11]. In 1997 much higher costs were estimated, with 4.5 billion dollars for direct and 3.4 billion dollars for indirect costs [6], while in 2001 about 10 billion dollars of indirect costs for AR were estimated, higher than the direct costs of the disease [12].

More recent surveys highlighted a further increase of the economic burden: a total of $\$ 6.1$ billion (in 2005 dollars) was spent on health care and treatment of allergic rhinitis in 2000 (excluding over-the-counter medications). By 2005, total expenditures to treat allergic rhinitis almost doubled to $\$ 11.2$ billion [8]. A 2003 European study reported an average annual cost of 1089 euros per child/adolescent and 1543 euros per adult [13]; indirect costs amounted to about $50 \%$ in adults but only to $6 \%$ in children, in whom nevertheless the calculation was based on time lost by parents/caregivers not including school absences.

Obviously, the costs further increase considering also asthma [14] For asthma, the estimated costs in Northern America in the 1990s amounted to 14 billion dollars $[15,16]$ and a recent study in the US calculated an average per person annual cost of asthma of 4912 dollars, of which 3180 due to direct costs - with drugs and hospitalizations as major contributors - and 1732 due to indirect costs [17].

Drug treatment accounts for a significant part of the direct and indirect costs of respiratory allergy. For example, first generation antihistamines (because of their sedating effects) impair mental performances more than untreated rhinitis does [18] and thus increase indirect cost, but recent generations antihistamines are more expensive and increase direct cost [19]. Also most drugs introduced in the latest decade to treat asthma (such as inhaled corticosteroids especially in association with long acting beta2-agonists) are more costly than the preceding agents. On the other hand, in a literature review lack of treatment, under treatment, or non-adherence were seen to increase both direct and indirect costs of allergic rhinitis [20]. In addition, no estimations of expenditure due to alternative medicine such as homeopathy, that is largely used, are available.

On this ground, any preventive strategy aimed at reducing the

${ }^{*}$ Corresponding author: Cristoforo Incorvaia, Allergy/Pulmonary rehabilitation Unit, ICP Hospital, Via Bignami, 120100 Milan, Italy, Tel: +39 02 5513852; Fax: +39 02 57993325; E-mail: cristoforo.incorvaia@gmail.com

Received November 01, 2011; Accepted January 18, 2012; Published January 23, 2012

Citation: Incorvaia C, Berto P, Ariano R, Elia R, Frati F (2012) Cost-Effectiveness of Allergen Immunotherapy. J Aller Ther S7:006. doi:10.4172/2155-6121.S7-006

Copyright: (c) 2012 Incorvaia C, et al. This is an open-access article distributed under the terms of the Creative Commons Attribution License, which permits unrestricted use, distribution, and reproduction in any medium, provided the original author and source are credited. 
clinical severity of respiratory allergy is potentially able to reduce its costs.

\section{Cost Effectiveness of Allergen Immunotherapy}

Allergen immunotherapy (AIT) is a treatment which reduces allergic symptoms by increasing tolerance to the specific allergen and modifying the natural history of the disease [21]. A number of studies showed that in the long term AIT is associated to a lower expenditure compared to drug treatment: a summary of the main studies of AIT and their characteristics and economic outcomes is presented in Table 1.

\section{Studies on subcutaneous immunotherapy}

The first studies conducted in Germany in the 1990s showed favorable results: Buchner and Siepe reported in a retrospective 10year analysis that direct and indirect costs in patients with allergic rhinitis and asthma were reduced by $54 \%$ in subjects treated with AIT compared to those treated with symptomatic drugs and estimated that in such 10-year period cost saving per patient should amounted to 9500 deutschmarks (DM) ( $€ 4860)$ for asthma and $5000 \mathrm{DM}(€$ 2560) for rhinitis [22]. In another study, Fischer estimated that use of subcutaneous AIT could save respectively 500 and 1000 DM (€ 256512) per year in subjects with AR and allergic asthma [23]. In the same years, data from the US showed that in patients with AR, AIT treatment reduced the cost of care by 180 dollars (circa $€ 140$ at current values) after 2 years of treatment, along with a significant improvement of quality of life [24] and that in patients with ragweed-induced asthma a cost reduction of about 30\% was reported during the performance of a placebo controlled study [25]. On the other hand, another study from the US on asthmatic patients found a mean cost about $20 \%$ higher in subjects who completed their AIT treatment versus non completers; however the same authors argued that the greater severity of asthma and consequently the higher drug use, in AIT completers may help explain such observation [26]. Moreover, the short duration of the study- 7 months- seems insufficient to achieve this cost reduction, which as we know from other studies, generally occurs after 2-3 years.

Another late 1990s German retrospective study analyzed the economic effects of 3 years of subcutaneous AIT modeling results over a follow-up of 10 years and found that advantages of AIT on drug treatment started after 6 years and resulted in final net savings of between 650 and $1190 \mathrm{DM}$ per patient (€ 332 and $€$ 608) [27]; a sensitivity analysis on direct medical costs showed that AIT was more likely to result in net savings than in additional costs. In this study the economic benefit was clear but the break-even point, that is the time at which the overall cost of treatment for AIT patients becomes lower than for patients receiving only drugs, was reached only 3 years after discontinuing AIT, while in a French study a significant reduction of the direct costs of allergic disease yet after 2 years of immunotherapy was reported [28].

In 2004, Bernstein by an economic modeling study found that over a 5-year period of observation the total cost per allergic patient was $\$$ 4560-4773 (€ 3507-3672) in subjects treated with AIT compared with \$ 10.200 (€ 7846) in subjects treated with standard drug treatment [29].

Three studies conducted in different European countries were published in recent years. In 2005, a health-economic analysis performed in Denmark on a large group of patients with grass pollen or mite allergy and including direct and indirect costs revealed that the direct cost per patient/year of standard care was respectively 2580 Danish kroner (DKK) (€ 347) per patient/year before AIT, and 1072 DKK ( $€ 144)$ after AIT [30]; in the long term, introduction of AIT was associated to additional direct costs of $13.676 \mathrm{DKK}(€ 1839)$ per patient, but when indirect costs were included in the economic evaluation AIT showed a positive net cost benefit of 1508 DKK (Eur 203). In 2006, an Italian study collected data on patients with AR and asthma caused by Parietaria pollen, who were treated with subcutaneous AIT by a Parietaria judaica extract by a conventional build-up schedule in 12 weeks and a maintenance treatment every 4 weeks for 3 years, or with antiallergic drugs [31]. Each patient was evaluated before starting AIT and then annually for 6 years in the pollen period of Parietaria by means of nose, eye and lung symptom scores and drug consumption as registered by diary cards. In other specifically designated cards the general practitioner's or specialist's visits, the number of desensitizing injections and the number of boxes of antiallergic drugs were registered.

A significant difference in favor of AIT plus drug treatment vs. drug treatment alone was observed, reaching a reduction of cost of about $15 \%$ at the $2^{\text {nd }}$ year and of $48 \%$ at the $3^{\text {rd }}$ year, with a highly statistical significance which was maintained up to the $6^{\text {th }}$ year, i.e. 3 years after stopping AIT, when a $80 \%$ reduction was found. The net saving for each patient at the final evaluation corresponded to 623 euros per year.

In a study conducted in France in 2007 a cost-effectiveness analysis was performed using a decision tree model in the perspective of the French Social Security, comparing AIT and current symptomatic treatment in adults and children with dust mite and pollen allergy [32] In adults, savings with subcutaneous AIT were $€ 393$ for dust mite and $€$ 1327 for pollen allergy over a 6-year period. In children, savings were $€$ 583 for dust mite and $€ 597$ for pollen allergy over a 7-year period. The

\begin{tabular}{|c|c|c|c|c|c|}
\hline Author (year) & Patients & AIT & Allergen & Study duration & Parameter/ Results \\
\hline Buchner (1995) (22) & adults & SCIT & pollen, mites & 10 years & $-54 \%$ costs for symptomatic treatment \\
\hline Schadlich (2000) (27) & adults & SCIT & pollen, mites & 10 years & Saving/patient 650-1190 DM (€ 332-608) \\
\hline Petersen (2005) (30) & adults & SCIT & pollen & 4 years & Saving/patient of 1508 DKK (€ 203) \\
\hline Ariano (2006) (31) & adults & SCIT & pollen & 6 years & - $48 \%$ money spent at year 4 \\
\hline Omnes (2007) (32) & adults \& children & SCIT & pollen, mites & 6 years & saving/patient $1327 €$ for pollen allergy, $393 €$ for mites \\
\hline Hankin (2008) (34) & children & SCIT & pollen, mites & 1,5 years & weighed 6-month saving/patient \$ 401 (€ 308) \\
\hline Hankin (2010) (35) & children & SCIT & pollen, mites & 1,5 years & - $34 \%$ total healthcare cost/patient \\
\hline Wang (2011) (36) & adults & SCIT & pollen, mites & 1,5 years & - $41 \%$ total healthcare cost/patient \\
\hline Berto (2005) (37) & children & SLIT & pollen, mites & 4 years & Saving/patient $2043 €$ \\
\hline Berto (2006) (38) & adults & SLIT & pollen & 6 years & Break-even at year 4 \\
\hline Bachert (2007) (40) & adults & SLIT & pollen & 9 years & Cost-effective for SLIT cost $<2200 € /$ year \\
\hline Canonica (2007) (41) & adults & SLIT & pollen & 4 years & Cost-effective for SLIT cost $<1900 € /$ year \\
\hline Berto (2008) (39) & adults & SLIT & pollen & 1 year & - $34 \%$ costs for symptomatic treatment \\
\hline Podladnikova (2008) (43) & adults & SLIT & pollen & 3 years & SLIT $684 €$ vs. SCIT $1004 €$ \\
\hline
\end{tabular}

Table 1: Economic studies of AIT. 
incremental cost-effectiveness ratio (ICER) was calculated and ranged from $€ 349$ (in children with dust mite allergy) to $€ 722$ (in adults with pollen allergy). The ICER is the most powerful measure of the value of a healthcare intervention in comparison with other interventions such as standard treatment or even placebo (no-treatment): the ICER is calculated as a ratio between the difference in costs (measured in the currency of interest such as Euro, Dollars etc.) and the difference in efficacy (measured in life years or as a percentage of patients who are successfully treated by the alternatives of interest). The final ICER value gives a measure of how much (in Euros or Dollars, etc.) is spent to gain one additional unit of efficacy [32].

In 2008, an economic modelling study in Germany on 1000 patients with AR or asthma receiving either AIT or standard drug treatment for 3 years over a time horizon of 15 years reported a total costs/patient of $€ 24.000$ with AIT vs. $€ 26.100$ with standard drug treatment; the resulting ICER was positive for all patients [33].

The most recent studies were conducted in the US. One study retrospectively analysed the Florida Medicaid claims data in children with a new diagnosis of AR; of 102,390 patients with new diagnoses of AR, 3048 (3\%) received AIT making it probably the largest economic study ever conducted on this topic [34]. Male patients, Hispanic patients and those with concomitant asthma were significantly more likely to receive IT. Approximately 53\% completed less than 1 year and $84 \%$ completed less than 3 years of IT. Patients who received IT used significantly less drugs, less outpatient visits and inpatient admissions and less resources in the 6 months after IT vs. before IT. Drugs ( $\$ 330$ vs. \$ 60 ) (€ 253 vs. $€ 46$ ), outpatient ( $\$ 735$ vs. $\$ 270$; $€ 565$ vs. $€ 207$ ) and inpatient ( $\$ 2441$ vs. $\$ 1 ; € 1878$ vs. $€ 0.77$ ) costs (including costs for AIT care) were significantly reduced after IT. The mean weighed 6-month saving was $\$ 401$ (€308). The authors concluded that despite suboptimal AIT duration (only $16 \%$ of patients completed 3 years of AIT), resource use and costs after treatment were significantly reduced from pre-AIT levels. A subsequent study used the same source of Florida Medicaid data to compare, over a time horizon of 10 years, the health care costs between 2 large groups of children with a new diagnosis of AR treated with subcutaneous AIT or with standard drug treatment, respectively [35]. The groups were matched by age at diagnosis of AR, sex, ethnicity and diagnosis of asthma and conjunctivitis. Children treated with AIT showed significantly lower 18 month median per-patient total health care costs ( $\$ 3247$ vs. $\$ 4872 ; € 2498$ vs. $€ 3748$ ), outpatient costs ( $\$ 1107$ vs. $\$ 2626$; $€ 852$ vs. $€ 2020$ ) and drug costs ( $\$ 1108$ vs. $\$ 1316$; $€ 852$ vs. $€$ 1012) compared with matched controls ( $\mathrm{p}<0.001$ for all comparisons). The significant difference in total health care costs was evident already 3 months after starting AIT and progressively increased through the end of the study. Similar results were obtained by the same Authors in another study on the Florida Medicaid claim database on 2089 adults $(0,7 \%$ of total registered population aged more than 18 years), recently diagnosed with AR: according to abstract data, at 18 months, costs for total healthcare services $(\$ 10,626$ versus $\$ 17,912 ; € 8173$ vs. $€ 13778$ $\mathrm{p}<0.0001$ ) as well as for each cost subgroup (inpatient, outpatient and pharmacy) all were significantly lower for patients in the SIT versus non-SIT group, with a global $41 \%$ healthcare cost reduction [36].

\section{Studies on sublingual immunotherapy}

The first published study, involving one Allergy center in Italy, dealt with the evaluation of cost effectiveness of sublingual immunotherapy (SLIT) in children with AR and asthma [37]. From records of pediatric patients seen for respiratory allergy, who had 1-year data prior to receive SLIT and 3-year data on high dose SLIT, outcome measures (the number of exacerbations, visits, absence from nursery or school) were analyzed. Both direct and indirect costs were considered. A second analysis compared a sub-group of children with allergic asthma, using a control group for costs, based on records of patients not treated with SLIT, extracted from a network-database of pediatricians.

An overall number of 135 patients were analyzed, 46 with perennial and 89 with seasonal allergy, with comparable gender and age distribution. A substantial reduction was found in all outcome measures (number of exacerbations, medical visits, absence from nursery or school) during SLIT compared to the previous period. The average annual cost/patient was $€ 2672$ before SLIT initiation and $€ 629$ / year during SLIT. Similar results were found for allergen subgroups. The asthma analysis involved 41 children with SLIT and 35 controls and also showed a substantial reduction in outcomes, though the direct cost per patient over the 4 years follow-up was $€ 1182$ for SLIT-treated children and $€ 1100$ for controls. These findings showed that high dose SLIT may be effective in reducing the global cost of allergic rhinitis and asthma and comparably expensive to conventional drug treatment in children with allergic asthma over a 4 year follow-up.

The second study analysed a cohort of adults with pollen allergy and was conducted using a decision tree developed and populated with epidemiologic and resource utilization data concerning about 2200 patients [38]. This study compared clinical outcomes, costs and produced cost/effectiveness ratios of SLIT associated with standard drug treatment versus standard drug treatment alone. The study was specifically designed for the Italian environment, based on a cost-effectiveness model originally developed for France [32] and considered the perspective of both the National Healthcare System (NHS) including only direct medical costs and the society, including both direct and indirect costs.

Drug therapy for AR and asthma was that recommended in international guidelines. Target population was young adults suffering from pollen-induced rhinitis with or without asthma. Time perspective was established at 6 years in order to include long term effectiveness of SLIT and patients' data were collected in 25 Italian centers. The study was based on the Retrospective Observation Physician Panel (ROPP), including 27 physicians from 25 allergy centers, who carried out data collection on epidemiology and consumption of resources, based on their internal medical database records. In particular, the physician panel provided information on total $\mathrm{AR}$ and/or asthmaduration and symptom improvement with-without SLIT; data collected were thus used to "populate" the economic model.

Main assessment criteria for the two different strategies were: a) costs, including direct medical costs assessed in the NHS perspective (visits, diagnostic procedures, drugs, SLIT and hospitalizations), as well as direct plus indirect costs (lost working days) and patient outof-pocket expenses, assessed in the societal perspective; $b$ ) effectiveness end-points, including: number of patients improved and number of asthma cases avoided; c) incremental cost per improved patient and incremental cost per asthma case avoided. The ICER was used to assess the difference between SLIT and no-SLIT based on their costs and effects.

The cost of SLIT was based on the dosing schedule recommended by the manufacturer. The number of follow-up visits per year by disease severity was retrieved from the ROPP and the relative cost $(20.66 €)$ was obtained from the National Outpatients Tariff List. The cost of diagnostic tests, according to international guidelines, was based on current NHS tariffs. Finally, the yearly number of hospital admissions was obtained from the ROPP data analysis and the cost of hospital 
admissions was based on current NHS tariffs.

The completed and returned questionnaires summarized the data of 2230 patients (age range $16-45$ year, mean age 28.2 years, $58 \%$ female). According to ROPP data analysis, $60.2 \%$ of patients had AR only and $39.8 \%$ AR with asthma. AR was severe in $31.9 \%$ and moderate in $68.1 \%$ of patients, whereas asthma was mild in $66.3 \%$ and moderate in 33.7 $\%$ of patients

A mean cost per patient treated over a period of 6 years was calculated for each therapeutic strategy and for each of the two perspectives studied. SLIT strategy resulted in less expense in term of both direct and indirect costs. The break-even point of SLIT, for the societal perspective, was reached at year 4 . This study showed that SLIT is more effective and less costly than no-SLIT from both the NHS and the societal perspective and these results remained stable over a realistic range of sensitivity analyses.

Another study evaluated the economics of SLIT in patients with pollen allergy and suffering from AR alone or associated with asthma compared with standard case controls [39]. This study was based on a longitudinal observational database operated by a network of Allergy centres. Patients were randomly assigned to SLIT (plus drugs as needed) or to treatment with drugs alone. The outcome measures included use of: drugs, SLIT, visits and laboratory tests. Costs were assessed in the perspective of the Italian NHS. Globally, 102 patients were evaluated. The overall per patient yearly cost of treatment was higher in SLIT patients, both in the whole sample ( $€ 311$ vs. $€ 180 /$ patient), in AR ( $€$ 288 vs. $€ 116)$ and AR associated with asthma ( $€ 362$ vs. $€ 230)$ subgroups. Patients with AR plus asthma generated more costs than AR alone in both groups. Nevertheless, considerable savings were obtained in the cost of symptomatic drugs $(-22 \%$ for $A R,-34 \%$ for $A R$ plus asthma) in SLIT patients, thus focusing the use of symptomatic drugs as an important indicator of effective allergy control. In general, efficacy studies provide evidence that SLIT can reduce the use of drugs, but this survey was the first to demonstrate this outcome in a routine care population, in the medical practice environment of an observational study and yet at the first year of treatment. It is of note that this study showed an inhaled corticosteroids sparing effect of SLIT.

Three recent studies were addressed on the evaluation of economic aspects of SLIT performed with oral tablets for grass pollen allergen in Northern Europe [40], Southern Europe [41] and the UK [42]. Aim of the first study was the assessment of cost-effectiveness of grass allergen tablets compared with use of symptomatic drugs in 7 Northern European countries. A societal perspective was adopted and the analysis had a 9-year time horizon. Main outcome measure was the Quality Adjusted Life Year (QALY). QALYs measure patients' healthrelated Quality of Life on a scale from 0 (death) to 1 (perfect health) and is a multi-attribute utility scale that can generate a single numeric index of health-related QoL.

The findings of this study showed that grass allergen tablet was clinically superior to symptomatic treatment, producing statistically significant differences for all efficacy end-points, including the number of QALYs gained (0.976 vs. 0.947). There was a significantly higher usage of rescue medications (antihistamines and corticosteroids) and more hours missed from work (productivity losses) in the symptomatic group [40]. The cost per QALY gained in the grass allergen tablet group was similar in the 7 countries ( $€ 12930$ to $€ 18263$ for an annual cost of the grass allergen tablet of $€ 1500$ ). The analysis showed that the grass allergen tablet was cost-effective for all countries for an annual cost below $€ 2200$.
This pharmacoeconomic analysis confirmed that SLIT is a costeffective intervention for the prevention of grass pollen induced rhinoconjunctivitis in Northern European countries, for a tablet price below $€ 6$. The second study assessed the cost-effectiveness of the same grass pollen oral tablets in patients suffering from grass pollen induced rhinitis living in four Southern European countries (Spain, France, Italy and Austria) [41]. A prospective pharmacoeconomic analysis was carried out alongside a multinational, clinical trial measuring the efficacy of grass pollen tablets. Pooled data on resource use and health outcomes were collected. A societal perspective was adopted and the analysis had a nine-year time horizon. The primary outcome measure was QALYs. SLIT was superior to standard care for all efficacy endpoints, including QALYs gained and resulted in significantly less use of rescue medication and fewer hours missed from work. Oral grass allergen tablet was cost-effective for all countries for an annual price in the range of $€ 1500-1900$. The result was improved by inclusion of future costs of asthma and exclusion of Spanish trial centres which experienced an exceptionally low pollen season.

The third study was conducted in the UK and was based on a prospective pharmacoeconomic analysis carried out as part of a multinational clinical trial assessing the efficacy of Grazax compared with placebo [42]. Both groups had access to symptomatic medication; thus the placebo group represented current standard care. Pooled data on health resource use, productivity loss because of absence from work and QALYs were collected in the trial. A societal perspective was adopted with a 9-year time horizon. The NHS price of Grazax of 2.25 pounds sterling $(€ 2.7)$ per tablet was used. The results showed that the QALY gain was significantly higher for patients receiving SLIT than for the placebo group receiving symptomatic medication alone $(0.197$ discounted QALYs gained 9 years into the future - equal to an extra 72 days of perfect health over 9 years). The resource use and productivity losses were higher for the placebo group. As a result, the cost per QALY gained with Grazax was 4319 pounds ( $€ 5176$ ), which is highly costeffective. Price analyses demonstrated that Grazax was cost-effective up to a tablet price of 5.07 pounds $(€ 6)$.

Also studies comparing the cost-effectiveness between subcutaneous AIT and SLIT are available. In the previously cited study by Omnes et al. concerning subcutaneous AIT [32], also SLIT was considered. In adults, the savings with subcutaneous IT were $393 €$ for dust mite and $1327 €$ for pollen allergy over a 6-year period. In children, the savings were $583 €$ for dust mite and $597 €$ for pollen allergy over a 7 -year period. The data from SLIT showed- as expected because of no need of visits for injections- higher savings, corresponding to $3158 €$ for dust mite and $1708 €$ for pollen allergy in adults and to $3938 €$ for dust mite and $824 €$ for pollen allergy in children.

Another study was done in Czech Republic on patients with allergic rhinoconjunctivitis receiving SLIT, SCIT, or only drugs for 3 years [43]. The total average direct cost per patient was $€ 416$ for SLIT and $€ 482$ for SCIT. A SLIT-treated patient paid less than a SCIT-treated patient for all out-of-pocket costs ( $€ 176$ vs. $€ 255)$ but paid more for allergen extracts ( $€ 72$ vs. $€ 55$ ). The sum of direct and indirect costs gave, over the 3 -year treatment, $€ 684$ for SLIT and $€ 1004$ for SCIT.

\section{Conclusions}

Among treatment options for respiratory allergy, AIT has unique characteristics, such as the capacity to change the natural history of the disease and, differently from drugs, to extend its effectiveness to many years after stopping therapy. Considering this, it is surprising that AIT is largely less used than drugs. For example, it has been reported that 
in the 1990s in Spain social expenditure for anti-asthmatic drugs rose by three times, while the expense for AIT was dwarfed to one third than that of the 1980s [44]. Indeed, there is an increasing mass of data indicating that both subcutaneous and sublingual AIT may be very beneficial to the healthcare system, in that either it could bring more clinical effectiveness at a reduced cost versus standard drug treatment, or it could bring extra benefit at an acceptable extra cost, thus fully accomplishing the significance of the term cost effectiveness. In particular, as stated by Hankin et al. in the conclusions of their study on health care cost benefits of AIT in children with AR "Greater use of this treatment in children could significantly reduce AR-related morbidity and its economic burden" [35].

The data reported by the numerous studies analyzed in this article, demonstrating a clear health economic advantage of AIT over drug treatment, should lead to carefully reconsider the optimal choice when deciding to treat a patient with AR or asthma.

\section{References}

1. The International Study of Asthma and Allergies in Childhood (ISAAC) Steering Committee (1998) Worldwide variation in prevalence of symptoms of asthma, allergic rhinoconjunctivitis and atopic eczema. Lancet 351: 1225-1232.

2. Sly RM (1999) Changing prevalence of allergic rhinitis and asthma. Ann Allergy Asthma Immunol 82: 233-248.

3. Upton MN, McConnachie A, McSharry C, Hart CL, Smith GD, et al. (2000) Intergenerational 20 year trends in the prevalence of asthma and hay feve in adults: the Midspan family study survey of parents and offspring. BMJ 321 : 88-92.

4. Linneberg A, Nielsen NH, Madsen F, Frolund L, Dirksen A, et al. (2000) Increasing prevalence of specific IgE to aeroallergens in an adult population: two cross-sectional studies 8 years apart; the Copenhagen Allergy Study. J Allergy Clin Immunol 106: 247-252.

5. Malone DC, Lawson KA, Smith DH, Arrighi HM, Battista C (1997) A cost of illness study of allergic rhinitis in the United States. J Allergy Clin Immunol 99 $22-27$

6. Mackowiak JI (1997) The health and economic impact of rhinitis. Am J Manag Care 3: S8-S18.

7. Ray NF, Baraniuk JN, Thamer M, Rinehart CS, Gergen PJ, et al. (1999) Direct expenditures for the treatment of allergic rhinoconjunctivitis in 1996, including the contributions of related airway illnesses. J Allergy Clin Immunol 103: 401407.

8. Anita Soni (2008) Medical Expenditure Panel Survey. Allergic Rhinitis: Trends in Use and Expenditures, 2000 and 2005.

9. Reed SD, Lee TA, McCrory DC (2004) The economic burden of allergic rhinitis. Pharmacoeconomics 22: 345-361.

10. Fineman SM (2002) The burden of allergic rhinitis: beyond dollars and cents Ann Allergy Asthma Immunol 88: 12-17.

11. McMenamin P (1994) Costs of hay fever in the United States in 1990. Ann Allergy $73:$ 35-39.

12. Kessler RC, Almeida DM, Berglund P, Stang P (2001) Pollen and mold exposure impairs the work performance of employees with allergic rhinitis. Ann Allergy Asthma Immunol 87: 289-295.

13. Schramm B, Ehlken B, Smala A, Quednau K, Berger K, et al. (2003) Cost of illness of atopic asthma and seasonal allergic rhinitis in Germany: 1-year retrospective study. Eur Respir J 21: 116-122.

14. O'Connell EJ (2004) The burden of atopy and asthma in children. Allergy 59 7-11.

15. Weiss KB, Sullivan SD (1996) Understanding the cost of asthma : the next step. CMAJ 154: 841-843.

16. Weiss KB, Sullivan SD (2001) The health and economics of asthma and rhinitis I. Assessing the economic impact. J Allergy Clin Immunol 107: 3-8.

17. Cisternas MG, Blanc PD, Yen IH, Katz PP, Earnest G, et al. (2003) A comprehensive study of the direct and indirect costs of adult asthma. J Allergy
Clin Immunol 111: 1212-1218.

18. Vuurman EF, van Veggel LM, Uitenwijk MM, Leutner D, O'Hanlon JF (1993) Seasonal allergic rhinitis and antihistamine effect on children's learning. Ann Allergy 71: 121-126.

19. Liao E, Leahy M, Cummins $G$ (2001) The costs of nonsedating antihistamine therapy for allergic rhinitis in managed care: an updated analysis. Am J Manag Care 7: S459-468

20. Schoenwetter WF, Dupclay L, Appayosyula S, Botterman MF, Pashos CL (2004) Economic impact and quality of life burden of allergic rhinitis. Curr Med Res Opin 20: 305-317

21. Bousquet J, Lockey RF, Malling HJ (1998) WHO Position Paper. Allergen immunotherapy: therapeutic vaccines for allergic diseases. Allergy 53: 4-30.

22. Buchner K, Siepe M (1995) Nutzen der Hyposensibilierung unter wirtschaftlichen Aspekten. Allergo J 4: 156-163.

23. Fischer P (1995) Therapie des allergisches Asthma. Die spezifische Hyposensibilierung kann helfen Kosten in Milliardenhohe einzusparen Laryngo-Rhino-Otologie 74: 28.

24. Kumar P, Kamboj S, Rao P (1997) The cost of care and quality of life in patients with allergic rhinitis on allergen immunotherapy. ACI Intern 9: 133-135.

25. Creticos PS, Reed CE, Norman PS, Khoury J, Adkinson NF, et al. (1996) Ragweed immunotherapy in adult asthma. N Engl J Med 334: 501-506.

26. Donohue J, Greneider DK, Connor-Lacke L, Canning CF, Platt R (1999) Utilization and cost of immunotherapy for allergic asthma and rhinitis. Ann Allergy Asthma Immunol 82: 339-347.

27. Schadlich PK, Brecht JG (2000) Economic evaluation of specific immunotherapy versus symptomatic treatment of allergic rhinitis in Germany. Pharmacoeconomics 17: 37-52.

28. Le Pen C, Rumeau-Pichen C, Lillin H (1997) L'impact de l'immunothérapie specifique sur le couts directs de la maladie allergique: une etude pragmatique. Rev Franc All Immunol Clin 37: 11-14.

29. Bernstein JA (2004) Pharmacoeconomic considerations for allergen immunotherapy. Clin Allergy Immunol 18: 151-164.

30. Petersen KD, Gyrd-Hansen D, Dahl R (2005) Health-economic analyse of subcutaneosus specific immunotherapy for grass pollen and mite allergy Allergol Immunopathol 33: 296-302.

31. Ariano R, Berto P, Tracci D, Incorvaia C, Frati F (2006) Pharmacoeconomics of allergen immunotherapy compared with symptomatic drug treatment in patients with allergic rhinitis and asthma. Allergy Asthma Proc 27: 159-163.

32. Omnes LF, Bousquet J, Scheinmann P, Neukirch F, Jasso-Mosqueda G, et al. (2007) Pharmacoeconomic assessment of specific immunotherapy versus current symptomatic treatment for allergic rhinitis and asthma in France. Eur Ann Allergy Clin Immunol 39: 148-156.

33. Bruggenjurgen B, Reinhold T, Brehler R, Laake E, Wiese G, et al. (2008) Costeffectiveness of specific subcutaneous immunotherapy in patients with allergic rhinitis and allergic asthma. Ann Allergy Asthma Immunol 101: 316-324.

34. Hankin C, Cox L, Lang D, Levin A, Gross G, et al. (2008) Allergy immunotherapy among Medicaid-enrolled children with allergic rhinitis: patterns of care resource use, and costs. J Allergy Clin Immunol 121: 227-232.

35. Hankin C, Cox L, Lang D, Bronstone A Fass P et al. (2010) Allergen immunotherapy and health care cost benefits for children with allergic rhinitis: a large-scale, retrospective, matched cohort study. Ann Allergy Asthma Immuno 104: 79-85.

36. Wang Z, Hankin CS, Cox L, Bronstone A (2011) Allergen Immunotherapy Significantly Reduces Healthcare Costs among U.S. Adults with Allergic Rhinitis: A Retrospective Matched Cohort Study Jointly Funded by the AAAA and ACAAI. The Journal of Allergy and Clinical Immunology 127: AB150.37

37. Berto P, Bassi M, Incorvaia C, Frati F, Puccinelli P, et al. (2005). Cost effectiveness of sublingual immunotherapy in children with allergic rhinitis and asthma. Eur Ann Allergy Clin Immunol 37: 303-308.

38. Berto P, Passalacqua G, Crimi N, Frati F, Ortolani C, et al. (2006) Economic evaluation of sublingual immunotherapy vs, symptomatic treatment in adults with pollen-induced respiratory allergy. The Sublingual Immunotherapy Pollen Allergy Italy (SPAI) study. Ann Allergy Asthma Immunol 97: 615-621. 
Citation: Incorvaia C, Berto P, Ariano R, Elia R, Frati F (2012) Cost-Effectiveness of Allergen Immunotherapy. J Aller Ther S7:006. doi:10.4172/21556121.S7-006

39. Berto P, Frati F, Incorvaia C, Cadario G, Contiguglia R, et al. (2008) Comparison of costs of sublingual immunotherapy and drug treatment in grass pollen induced allergy: results from the SIMAP database study. Curr Med Res Opin 24: 261-266.

40. Bachert C, Vestenbaek U, Christensen J, Griffiths UK, Poulsen PB (2007) Costeffectiveness of grass allergen tablet (Grazax) for the prevention of seasonal grass pollen induced rhinoconjunctivitis- a Northern European perspective. Clin Exp Allergy 37: 772-779.

41. Canonica GW, Poulsen PB, Vestenbaek U (2007) Cost-effectiveness of Grazax for prevention of grass pollen induced rhinoconjunctivitis in Southern Europe. Respir Med 101: 1885-1894.
42. Nasser S, Vestenbaek U, Beriot-Mathiot A, Poulsen PB (2008) Costeffectiveness of specific immunotherapy with Grazax in allergic rhinitis coexisting with asthma. Allergy 63: 1624-1629.

43. Podladnikova J, Krcmova I, Vicek J (2008) Economic evaluation of sublingual vs. subcutaneous allergen immunotherapy. Ann Allergy Asthma Immunol 100 482-489.

44. Nieto A, Alvarez-Cuesta E, Boquete M, Mazon A, de la Torre F (2001) The cost of asthma treatment in Spain and rationalizing the expense. J Investig Allergol Clin Immunol 11: 133-148.
This article was originally published in a special issue, Pulmonary developmental biology handled by Editor(s). Dr. Rodolfo de Paula Vieira, University Hospital Freiburg, German 